\title{
Escala de Bem-Estar Afetivo no Trabalho (Jaws): Evidências de Validade Fatorial e Consistência Interna
}

\author{
Job-Related Affective Well-Being Scale (Jaws): Evidences \\ of Factor Validity and Reliability
}

\author{
Valdiney Veloso Gouveia*, Patrícia Nunes da Fonsêca, Samuel Lincoln Bezerra Lins, \\ Aline Vieira de Lima \& Rildésia S. V. Gouveia \\ Universidade Federal da Paraíba, João Pessoa, Brasil
}

\begin{abstract}
Resumo
O objetivo deste estudo foi adaptar uma medida de bem-estar afetivo no trabalho para o contexto brasileiro. Especificamente, pretendeu-se conhecer evidências de validade fatorial e consistência interna da Job-Related Affective Well-Being Scale (JAWS), avaliando se as pontuações nos seus fatores diferem em função do gênero e da idade dos participantes. Participaram 298 trabalhadores de centros comerciais de pequeno e médio porte da cidade de João Pessoa (PB). A maioria destes era do sexo feminino (76,8\%), com idade média de 26 anos (DP $=6,87$ ). Através de uma análise dos componentes principais (rotação promax) foram identificados dois fatores que explicaram conjuntamente $48,1 \%$ da variância total: afetos positivos ( $\alpha=0,94 ; 14$ itens) e afetos negativos ( $\alpha=0,87 ; 13$ itens); um fator geral de bem-estar afetivo no trabalho foi também computado $(\alpha=0,95 ; 27$ itens). As pontuações dos participantes nestes fatores não foram influenciadas pelas variáveis gênero e idade. Estes resultados são discutidos à luz do que tem sido escrito sobre os parâmetros desta escala e da relação dos afetos com estas variáveis demográficas.

Palavras-chave: Afetos; emoções; bem-estar; trabalho.
\end{abstract}

\begin{abstract}
This study aimed at adapting a measure of job-related affective well-being for the Brazilian milieu. Specifically, it was proposed to know evidences of factor validity and reliability of the Job-Related Affective WellBeing Scale (JAWS), assessing if its scores are influenced by participants' gender and age. The participants were 298 individuals employed in small or middle shopping malls in the city of João Pessoa, PB; most of them were female $(76.8 \%)$, with a mean age of 26 years old $(S D=6.87)$. A main component analysis (with promax rotation) was performed, revealing two components that jointly accounted for $48.1 \%$ of the total variance. They were named as positive affect $(\alpha=.94 ; 14$ items) and negative affect ( $\alpha=.87 ; 13$ items). A general factor of affective well-being was also identified ( $\alpha=.95 ; 27$ items). Participants' scores on these factors were not influenced by their gender or age. These findings are discussed based on literature that describes the psychometric parameters of the JAWS as well as the correlation of affects with demographic variables.

Keywords: Affects; emotions; well-being; work.
\end{abstract}

Nos últimos anos, a saúde mental do trabalhador tem sido temática de interesse na Psicologia Social e Organizacional, visto as transformações ocorridas no mundo do trabalho no final do século XX e início do presente (Álvaro, 1992; T. L. Carneiro \& Carvalho, 2003). No mercado de trabalho, especificamente, assiste-se a uma diversidade de formas de emprego, sobretudo com a expansão das relações de trabalho temporário. Neste cenário, observa-se, por exemplo, que as pessoas que trabalham há menos de dois

\footnotetext{
* Endereço para correspondência: Universidade Federal da Paraíba, Centro de Ciências Humanas Letras e Artes, Campus I, Departamento de Psicologia, Cidade Universitária, s/n, João Pessoa, PB, 58051-900. Tel.: (83) 32167337 ; Fax: (83) 3216 7064. E-mails: vvgouveia@uol.com.br, patynfonseca@hotmail.com e rildesia.val@gmail.com
}

anos são mais susceptíveis a doenças e acidentes de trabalho do que a média dos trabalhadores. Este padrão é extensivo aos empregados temporários, principalmente quando são de setores da construção civil, saúde e de atividades sociais (Comissão de Comunidades Européias, 2002). Contudo, tal panorama modifica-se quando as pessoas têm estabilidade no emprego, pois, segundo Evans e Steptoe (2002), a situação de permanência no trabalho possibilita a obtenção de direitos trabalhistas e a segurança de que suas necessidades básicas podem ser satisfeitas, proporcionando ao trabalhador mais bem-estar geral.

$\mathrm{Na}$ linha do que se comentou previamente, Mäkikangas e Kinnunem (2003) enfatizam que nas duas últimas décadas o trabalhador tem passado por mudanças laborais que têm aumentado seu nível de estresse no trabalho e decrescido o de bem-estar. No que diz respeito à sua saúde, a 
Psicologia já vem efetivamente dando sua contribuição quando estuda os fatores de risco e as formas de melhoria das condições de trabalho (Álvaro, 1992; Sauter \& Hurrel Jr., 1999). Não obstante, esta disciplina não tem se limitado a estes aspectos; os pesquisadores da área têm ampliado seus horizontes, dando atenção igualmente à temática do bem-estar subjetivo, enfocando como as emoções positivas e negativas, por exemplo, podem afetar a saúde do trabalhador (Carter, 2004; Cheng, 2004; Jamal, 1999; Sevastos, Smith, \& Cordery, 1992; Zapf, 2002). Este aspecto compreende o interesse principal deste artigo, demandando-se considerar o conceito de bem-estar subjetivo e conhecer como este se insere no contexto do trabalho.

\section{Bem-estar Subjetivo: Conceituação e Papel no Contexto Laboral}

Os estudos sobre o bem-estar tiveram começo nos anos de 1960 (Diener, 2000; Diener, Suh, Lucas, \& Smith, 1999) e, desde então, tem havido um crescente interesse por parte dos pesquisadores (Albuquerque \& Tróccoli, 2004). Este construto tem recebido diversas denominações, tais como felicidade, satisfação, afetos positivos e avaliação subjetiva da qualidade de vida (Diener et al., 1999). Contudo, assume-se aqui a denominação geral de bem-estar afetivo.

Para Ryan e Deci (2001) o bem-estar é um estado de satisfação do indivíduo com ele mesmo e com o seu meio. Refere-se ao que as pessoas pensam e como elas se sentem sobre suas vidas. Com isso, pode-se dizer que o bem-estar é constituído por dois componentes principais: o cognitivo e o afetivo. O primeiro é conhecido como satisfação com a vida, e permite que as pessoas façam uma avaliação global de sua própria vida; já o segundo envolve os componentes emocionais e permite a pessoa vivenciar emoções de prazer (positivas) e desprazer (negativas) (Diener, Suh, \& Oishi, 1997).

As emoções têm tido um papel de destaque nas pesquisas com seres humanos, com especial destaque para a Psicologia (Carter, 2004). Este construto é expresso através dos afetos positivos e negativos, compreendendo um elemento central na definição do bem-estar subjetivo (Gouveia et al., 2003). Porém, o fato de um indivíduo apresentar um alto nível de bem-estar não significa necessariamente ausência de emoções negativas, mas que as emoções positivas sobrepujam as negativas (Albuquerque \& Troccóli, 2004).

Em anos recentes, em razão do aumento do número de empregados com problemas de saúde, tem crescido o montante de empresas que buscam programas de prevenção de doenças e de promoção da saúde e do bem-estar dos seus empregados (Donaldson \& Blanchard, 1995). Esta questão passou a chamar a atenção dos pesquisadores, que passaram a tomar as emoções como indicadores de tensão e bem-estar no trabalho (ver, por exemplo, Daley \& Parfitt, 1996; Jamal, 1999; Sevastos et al., 1992; Wright \& Doherty, 1998). Neste sentido, prontamente buscaram compreender até que ponto as emoções vivenciadas pelos indivíduos em decorrência do trabalho afetam sua saúde e seu desempenho profissional.

No geral, tem-se constatado que as emoções, especialmente aquelas advindas do trabalho, podem gerar tanto efeitos positivos quanto negativos à saúde do trabalhador. Pessoas que freqüentemente sofrem de exaustão emocional, ansiedade e depressão no trabalho, mostram-se insatisfeitas com suas vidas e não conseguem desempenhar a contento suas atividades profissionais (Carter, 2004; Cheng, 2004; Zapf, 2002).

De acordo com Kirschenbaum, Oigenblick e Goldberg (2000), as emoções negativas, resultantes de uma humilhação no trabalho, por exemplo, impedem o indivíduo de gozar de bem-estar, ao mesmo tempo em que o deixa predisposto a acidentes de trabalho, especialmente se os locais de trabalho apresentam periculosidade. Diversos estudos demonstraram que os trabalhadores que exercem funções diretamente com o público ou que têm um alto grau de exigência estão mais propensos a apresentarem exaustão emocional, queixas de sintomas físicos e psicossomáticos, assim como insatisfação com o trabalho (Jorge, Bosma, Peter, \& Siegrist, 2000; Zapf, 2002).

Estudando a relação entre o ambiente externo e o trabalho, Herzberg (1959) alicerçou sua teoria dos dois fatores. Segundo esta teoria, a satisfação e a insatisfação no trabalho constituem dimensões separadas, onde a ausência de um elemento não implica necessariamente a presença do outro. Neste caso, a ausência de determinados fatores de trabalho (por exemplo, salário, benefícios sociais, políticas da empresa, clima de relações entre a direção e os empregados) pode acarretar insatisfação; entretanto, a sua presença não causaria satisfação, mas evitaria a insatisfação. Consoante com este autor, é no ambiente de trabalho que o indivíduo procura condições de satisfazer as suas necessidades (fisiológicas, de segurança, sociais, de estima e de auto-realização) e assim obter o bem-estar mínimo necessário para desenvolver suas atividades laborais com eficácia e prazer.

É na busca do bem-estar que as pessoas percebem que o trabalho atende o desejo humano, correspondendo à condição de ser membro produtivo da sociedade ao mesmo tempo em que este as direciona para um objetivo na vida (Morin, 2001). Neste caso, o trabalho contribui criando certa medida de auto-estima, saúde mental e convívio social (Álvaro, 1992; Porto \& Tamayo, 2003). Por outro lado, há aqueles trabalhos em que as pessoas sofrem pressões, convivem com constantes situações de fracasso e ameaças de desemprego. Nestes casos, as necessidades dos indivíduos não são satisfeitas, havendo um aumento progressivo de emoções negativas chegando a ponto de os trabalhadores perderem a satisfação com o que fazem e não gozarem de bem-estar (Kirschenbaum et al., 2000).

Parece evidente, pois, que ter em conta o bem-estar pode ser importante para explicar e compreender a satisfação que as pessoas têm com seu trabalho. Pode igualmente ser útil para estimar o seu desempenho e potencial envolvimento em acidentes laborais. Deste modo, constitui-se etapa fundamental contar com instrumentos psicometricamente 
adequados para avaliar o bem-estar afetivo no trabalho. Em busca realizada na Biblioteca Virtual em Saúde (2006), foram encontrados 19 artigos em que tinha sido empregado ao menos um dos seguintes termos (freqüência entre parênteses): "emoções no trabalho" (dez), "afetos no trabalho" (seis) e "bem-estar no trabalho" (três). Não obstante, nenhum tratou especificamente de ins-trumentos de medida. Apesar deste aspecto, foi possível localizar no contexto estadunidense uma escala construída recentemente cujo propósito é justamente avaliar o bem-estar afetivo relacionado ao trabalho (Katwyk, Spector, Fox, \& Kelloway, 2000). O presente artigo tem como objetivo principal adaptá-la para uso no Brasil, o que demanda inicialmente descrevê-la.

\section{Escala de Bem-Estar Afetivo no Trabalho}

A Job-related Affective Well-being Scale (JAWS) foi inicialmente proposta por Katwyk et al. (2000), compreendendo uma variedade de emoções identificadas como positivas ou negativas no ambiente de trabalho. Esta foi elaborada a partir de três estudos realizados nos Estados Unidos, os quais são resumidos a seguir:

Estudo 1. Procurou-se conhecer a estrutura dos afetos relacionados ao trabalho, contrapondo-lhes com os afetos em geral. A partir de uma lista de 235 afetos, três dos autores da escala procuraram avaliá-los em termos de se (a) diziam respeito ao bem-estar afetivo, e (b) poderiam ser incluídos sob a referência de "meu trabalho me faz sentir...". Houve consenso final ( sic) de que 56 dos afetos cumpriam os critérios de inclusão. Este conjunto de afetos, cada um escrito em um cartão, foi apresentado a 51 estudantes universitários empregados, com idade média de 22 anos (amplitude de 18 a 45), a maioria mulheres (72,5\%). Sua tarefa consistia em reunir em blocos os afetos que eram mais similares, seguindo as etapas que se descrevem: (a) inicialmente, deveriam organizar livremente os afetos em tantos blocos como julgassem necessários; e, (b) subseqüentemente, deveriam distribuir os afetos em 4, 8 e 16 blocos. Isso permitiu criar uma medida de similaridade entre cada par de estímulos (afetos), ponderada pelo número de categorias usadas. Com base nesta medida, criou-se uma matriz de similaridade de 55 afetos (o item "meu trabalho me faz sentir exasperado" foi eliminado, pois cerca da metade dos participantes não compreendeu seu significado) e procedeu-se a um escalonamento multidimensional (ALSCAL), fixando a solução em duas dimensões. Os índices de ajuste foram favoráveis $(R S Q=0,94$ e Stress $=0,14)$. Esta revelou que os estímulos podem ser organizados em uma dimensão de prazer-desprazer e outra que evidencia o grau de excitação (baixo vs. alto), corroborando o modelo bidimensional de bem-estar afetivo relacionado ao trabalho, como o propôs Warr (1987).

Estudo 2. Este teve por objetivo validar as dimensões de prazer-desprazer e baixa-alta excitação, como evidenciada no estudo anterior, produzindo a versão preliminar da Escala de Bem-estar Afetivo no Trabalho (JAWS). Parti- ciparam 100 estudantes universitários empregados, com média de idade de 23 anos (amplitude de 18 a 45); nenhuma informação foi dada quanto ao sexo. O tempo médio de trabalho destes participantes foi de 21,9 meses (amplitude de 1 a 256 meses), tendo sido relatados 55 diferentes tipos de trabalho (por exemplo, professores, técnico de laboratório, gerente de vendas). Os participantes deveriam responder duas versões da escala, composta pelos 55 afetos resultantes do estudo anterior: uma em que cada um dos afetos foi avaliado de acordo com o prazer vs. desprazer e outra em que se avaliou o grau de excitação (baixo vs. alto). Procurou-se contrabalanceá-las, designando aleatoriamente os participantes para responder uma ou outra versão em primeiro lugar. A correlação das pontuações dos 55 afetos relacionados ao trabalho quando respondidos em termos de (des)prazer e excitação foi de 0,48 ( $p<0,01)$; além disso, foram significativas $(p<0,01)$ as correlações para os 22 afetos de prazer $(r=0,41)$ e os 33 de desprazer $(r=0,48)$

Estudo 3. Os dois estudos anteriores buscaram apoiar a suposição de Warr (1987) de que o afeto relacionado ao trabalho expressa duas dimensões: prazer e excitação. No presente estudo o propósito foi conhecer o quanto a $J A W S$ se correlacionava com medidas de satisfação com o trabalho e traços afetivos (validade convergente-discriminante) e a consistência interna (Alfa de Cronbach) das suas dimensões. Participaram 114 empregados da Universidade do Sul de Flórida. A idade média destes foi de aproximadamente 42 anos (amplitude de 23 a 65), a maioria mulheres (82\%). Neste caso, empregou-se uma versão da JAWS com 30 itens, igualmente distribuídos entre afetos positivos e negativos. Estes foram selecionados da lista original de 56 itens, procurando eliminar redundâncias e considerar unicamente aqueles itens que refletissem estados afetivos em relação ao trabalho. Além deste instrumento, os participantes responderam as seguintes medidas: (a) a Positive Affectivity-Negative Affectivity Scale (PANAS), com 20 itens igualmente distribuídos para medir afetos positivos e negativos (Watson, Clark, \& Tellegen, 1988), (b) três itens que avaliavam satisfação com o trabalho, tendo sido retirados da Michigan Organizational Assessment Scale (Camman, Fishman, Jenkins, \& Klesh, 1979), (c) os três itens da sub-escala de autonomia da Job Diagnostic Survey (Idaszak \& Drasgow, 1987), (d) três escalas para avaliar estressores no trabalho (Spector \& Jex, 1998): Quantitative Workload Inventory (cinco itens), Interpersonal Conflict at Work Scale (quatro itens) e Organizational Constrains Scale (onze itens) e (e) duas medidas de tensão no trabalho: um item para avaliar o quanto o respondente pensou seriamente em deixar o seu trabalho atual, e os 18 itens que compõem o Physical Symptom Inventory (Spector \& Jex, 1998), que descreve sintomas físicos de saúde que este tem sentido nos últimos 30 dias. Como previsto, a JAWS se correlacionou positiva e significativamente $(p<0,05)$ com afetos positivos $(r=0,61)$, satisfação com o trabalho $(r=$ $0,73)$, autonomia $(r=0,21)$, tendo-o feito negativamente com afetos negativos $(r=-0,53)$, carga laboral $(r=-0,23)$, restrição organizacional $(r=-0,39)$, conflito interpessoal 
$(r=-0,34)$, desejo de deixar o trabalho $(r=-0,60)$ e sintomas físicos $(r=-0,33)$. Ainda neste estudo foi comprovada a consistência interna (Alfa de Cronbach) da JAWS total, que foi de 0,95 .

Em resumo, o conjunto destes estudos permitiu reunir evidências em favor da validade de construto e convergente e da precisão deste instrumento. Não obstante, nenhuma informação foi apresentada acerca da sua estrutura fatorial, carecendo também de evidências sobre sua adequação em outra cultura, uma vez que os estudos de Katwyk et al. (2000) se limitaram ao contexto estadunidense.

Tendo em conta estes aspectos, Rode (2005) decidiu checar a adequação da JAWS em uma amostra eslovena. No caso, aplicou a versão de 30 itens deste instrumento a 96 trabalhadores que atuavam em centros de serviço social, a fim de conhecer a estrutura fatorial resultante e os índices de consistência interna dos respectivos fatores. Em síntese, considerou para análise a versão completa e uma reduzida, em que saíram os cinco seguintes itens: tranqüilo, incomodado, orgulhoso, animado e empolgado. Para ambas as versões, foi possível identificar uma estrutura com dois fatores, agrupando separadamente os itens de afetos positivos e negativos, explicando $38,3 \%$ (42,2\% quando os cinco itens problemáticos foram eliminados) da variância total. Unicamente para a versão completa foram apresentados os Alfas de Cronbach, tendo o fator geral se situado em 0,89 (30 itens) e os específicos em 0,89 (afetos negativos) e 0,85 (afetos positivos).

Como é possível perceber, o estudo de Rode (2005) reforça a concepção de ser possível identificar claramente os afetos positivos como distintos dos negativos (Katwyk et al., 2000; Warr, 1987; Watson et al., 1988). Contudo, ressalta-se que sua amostra foi pequena $(N<100)$ e homogênea (trabalhadores de um único centro, serviço). Portanto, caberia seguir conhecendo evidências de sua estrutura fatorial, considerando uma amostra mais ampla e diversa em termos de características demográficas, uma vez que estas podem influenciar as pontuações dos participantes em medidas de bem-estar, como se discute a seguir.

\section{Bem-estar Subjetivo e Variáveis Demográficas}

De acordo com Albuquerque e Troccóli (2004), as condições externas ao indivíduo (por exemplo, riqueza, saúde física e local de atividade laboral) podem influenciar na determinação dos afetos, todavia não os define. A subjetividade, ou seja, as experiências internas de cada um, estas sim, são as principais definidoras das emoções, dos afetos e do bem-estar. Neste marco, é comum procurar explicar o bem-estar subjetivo a partir de atributos psicológicos que descrevem as pessoas, como seus traços de personalidade (Schiffman, Reynolds, \& Young, 1981), interesses vocacionais (Gusmão, 2004), valores humanos (Chaves, 2003) ou mesmo suas emoções (Cheng, 2004; Katwyk et al., 2000). Contudo, existem evidências de sua variação em detrimento das características demográficas das pessoas (Christopher, Kuo, Abraham, Noel, \& Linz, 2004; Clark, 1997; Otta \& Fiquer, 2004; Ryff, 1989).
A variável gênero tem sido relacionada ao bem-estar subjetivo e aos afetos. A propósito, em diversos estudos têm se verificado que os homens apresentam um nível mais elevado de bem-estar subjetivo do que as mulheres (Caetano \& Estrada, 2006; Chaves, Gouveia, Gusmão, Santos, \& Araújo, 2002). De modo semelhante, Hutchinson et al. (2004) constataram que as mulheres apresentam níveis mais baixos de bem-estar psicológico e satisfação com a vida do que os homens. Pode-se observar nas mulheres maior presença de estados de depressão, ansiedade e mais afetos negativos (Fujita, Diener, \& Sandvik, 1991), embora estas demonstrem nível de felicidade igual ou inclusive superior ao dos homens. Peiró (2006) ressalta que em alguns países (por exemplo, Austrália, China, Finlândia, Japão, Nigéria e Sudão) as mulheres declararam um grau maior de felicidade geral do que o fizeram os homens.

Analisando o papel da variável idade do trabalhador em relação ao seu nível de bem-estar subjetivo e exaustão emocional, Santavirta, Kovero e Soloviera (2005) encontraram que professoras e enfermeiras, com idades mais avançadas, percebiam o trabalho como uma atividade que não exigia muito esforço, diferentemente das trabalhadoras mais jovens que o percebiam como uma atividade que demandava muito empenho, a ponto de causar-lhes exaustão emocional e, assim, comprometer o seu bem-estar. Neste caso, constatou-se que a percepção do trabalho pelas funcionárias de diferentes idades contribuiu de forma decisória na avaliação do seu bem-estar. Porém, isso se evidenciou, principalmente, em empresas que têm exigido maior esforço mental e mais tempo de dedicação ao trabalho por parte dos empregados. Outros estudos têm também constatado que as pessoas mais idosas apresentam um nível de bem-estar maior do que aquelas mais jovens (Otta \& Fiquer, 2004; Ryff, 1989). Neste caso, a relação entre idade e bem-estar sugere que, com o avanço da idade, as emoções parecem ser mais bem reguladas, ou seja, os indivíduos já conseguem maximizar os afetos positivos e minimizar os negativos, fato que promoveria maior adaptação aos eventos da vida e proporcionaria sentimentos de bem-estar.

Em resumo, esta pesquisa procura adaptar para o contexto brasileiro a Escala de Bem-Estar Afetivo no Trabatho, reunindo evidências acerca de sua validade fatorial e consistência interna. Pretendeu-se ainda conhecer em que medida suas pontuações podem ser explicadas por duas variáveis de natureza demográfica: gênero e idade. Como assinalam Katwyk et al. (2000), este instrumento reúne algumas vantagens em relação a outros existentes na literatura. Por exemplo, (a) centra-se na emoção pura e simplesmente, ao invés de deter-se em crenças e satisfação no trabalho; (b) interessa-se pelo contexto afetivo específico ao trabalho, divergindo de medidas como a Escala de Afetividade Positiva e Negativa (PANAS) (Watson et al., 1988), que avalia emoções em contextos livres; e (c) cobre uma gama extensa de respostas afetivas. Estes aspectos parecem reforçar a pertinência da presente pesquisa. 


\section{Método}

\section{Amostra}

Participaram deste estudo 298 pessoas que trabalhavam como atendentes ao público em centros comerciais de pequeno e médio porte na cidade de João Pessoa - PB. A idade variou de 15 a 64 anos $(M=25,8 ; D P=6,87)$, sendo a maioria do sexo feminino $(76,8 \%)$, tendo concluído o ensino médio $(69,8 \%)$ e exercendo a função de vendedor $(65,2 \%)$. Com relação ao tempo de trabalho na empresa, a maioria indicou ter um $(38,9 \%)$ ou dois $(22,1 \%)$ anos; os que afirmaram seguir na empresa por mais de cinco anos compreenderam cerca da quinta parte do total (22,5\%). A maioria se auto-percebia como de classe média $(77,8 \%)$.

\section{Instrumento}

Os participantes receberam uma folha impressa frente e verso, constando da Escala de Bem-estar Afetivo no Trabalho - JAWS (Katwyk et al., 2000) e seis perguntas demográficas: idade, sexo, escolaridade, função, tempo que trabalha na empresa e classe sócio-econômica percebida. No caso, o participante poderia indicar uma de três alternativas: baixa, média ou alta.

A JAWS descreve as reações emocionais dos respondentes no contexto do trabalho, compreendendo 30 itens (por exemplo, Meu trabalho me faz sentir tranqüilo; Meu trabalho me faz sentir com raiva; Meu trabalho me faz sentir alegre), respondidos em escala de cinco pontos, variando de 1 (Nunca) a 5 (Sempre). Informações acerca dos seus parâmetros psicométricos foram apresentadas previamente, quando se detalhou sua elaboração e validação. Um psicólogo bilíngüe fez sua tradução do inglês para o português, sendo esta posteriormente revisada por uma professora do curso de Letras com habilitação em Língua Inglesa. Seguiu-se, finalmente, o método de back-translation, em que a última versão traduzida para o português foi retraduzida para o inglês, contando com a ajuda de um terceiro colaborador, também bilíngüe; comparando as versões original e retraduzida, atestaram-se suas equivalências.

Posteriormente, procedeu-se à validação semântica da $J A W S$. Na oportunidade participaram onze pessoas, que tinham idades entre 18 a 65 anos. A escolaridade variou do ensino fundamental incompleto até o nível superior. Esta validação permitiu verificar se os itens eram legíveis para a parcela com menor escolaridade da população-alvo, bem como averiguar se a linguagem utilizada também era adequada à amostra com o nível educacional mais elevado. Nesta etapa do estudo, com vistas a tornar os itens mais compreensíveis para os participantes, foram feitas modificações nas expressões usadas em sete itens (1, 5, 14, 19, 24,28 e 30), bem como foi simplificado o texto de instrução de como responder a escala. O leitor interessado poderá solicitar uma cópia da versão atual deste instrumento.

\section{Procedimento}

Após autorização dos administradores e/ou proprietários dos estabelecimentos, os funcionários foram convidados a participar da pesquisa. Em caso de recusa, buscava-se outra pessoa que aceitasse participar. Neste ensejo, era-lhe garantido o anonimato e o sigilo das respostas. A aplicação foi realizada de forma individual no próprio ambiente de trabalho, contando com a colaboração de quatro estudantes de graduação em Psicologia, que receberam treinamento prévio acerca de como proceder. Neste caso, foram instruídos a prestar esclarecimentos apenas sobre a forma de resposta, nunca com relação ao conteúdo do instrumento. Em média dez minutos foram suficientes para completar sua participação.

\section{Análise de Dados}

Inicialmente, foi realizada a comprovação do poder discriminativo dos itens através de um teste convencional de comparação de médias ( $t$ de Student). Neste caso, formaram-se os grupos inferior e superior a partir da pontuação total mediana; o item poderia ser considerado discriminativo se estes grupos apresentassem pontuações diferentes $(p<0,05)$ e na direção teoricamente esperada. A fim de verificar a estrutura fatorial da escala, os dados foram submetidos a uma análise de Componentes Principais $(P C)$, empregando-se a rotação promax. Uma sintaxe escrita para o SPSS foi empregada para efetuar a análise paralela. Depois de determinada a dimensionalidade da escala, calculou-se um Alfa de Cronbach (a) para cada componente resultante, checando sua consistência interna e homogeneidade. Empregaram-se MANOVAs para testar o efeito das variáveis idade e gênero nos fatores de afetos no trabalho.

\section{Resultados}

\section{Poder Discriminativo dos Itens}

Como antes especificado, o poder discriminativo dos itens foi estabelecido através de grupos critérios internos, considerando os participantes cujas pontuações totais se localizaram abaixo e acima do ponto mediano, chamando-os, respectivamente, de grupo inferior e superior. De acordo com o critério assumido (isto é, $p<0,05$ ), nenhum item foi eliminado do conjunto original, ou seja, todos diferenciaram os respondentes com magnitudes próximas. Uma tabela completa com estes resultados está disponível, sob solicitação, a um dos autores do estudo.

\section{Evidências de Validade e Precisão (Consistência Interna)}

Inicialmente, confirmou-se a fatorabilidade da matriz de correlação entre os itens da escala através do $K M O=0,94$ e o Teste de Esfericidade de Bartlett, $\mathrm{c}^{2}(435)=4579,17 ; p$ $<0,001$ (ver Tabachnick \& Fidel, 2001). Efetuada uma $P C$, sem fixar número de componentes a extrair ou tipo de rotação, foi possível encontrar até quatro componentes que atenderam o critério de Kaiser, isto é, valor próprio (eigenvalue) igual ou superior a 1. Estes explicaram conjuntamente $56,4 \%$ da variância total. Contudo, devido a este critério superestimar o número de componentes a extrair, decidiu-se tomar em conta outros dois critérios mais 
Gouveia, V. V., Fonsêca, P. N., Lins, S. L. B., Lima, A. V. \& Gouveia, R. S. V. (2008). Escala de Bem-Estar Afetivo no Trabalho (Jaws): Evidências de Validade Fatorial e Consistência Interna.

robustos (Hayton, Allen, \& Scarpello, 2004): a distribuição gráfica dos valores próprios (Cattell) e, principalmente, a geração aleatória de tais valores (Análise Paralela). Neste caso, assumiram-se os parâmetros do banco de da- dos original (298 participantes e 30 variáveis), realizando 1.000 simulações. Os resultados correspondentes são sumarizados na Figura 1 a seguir.

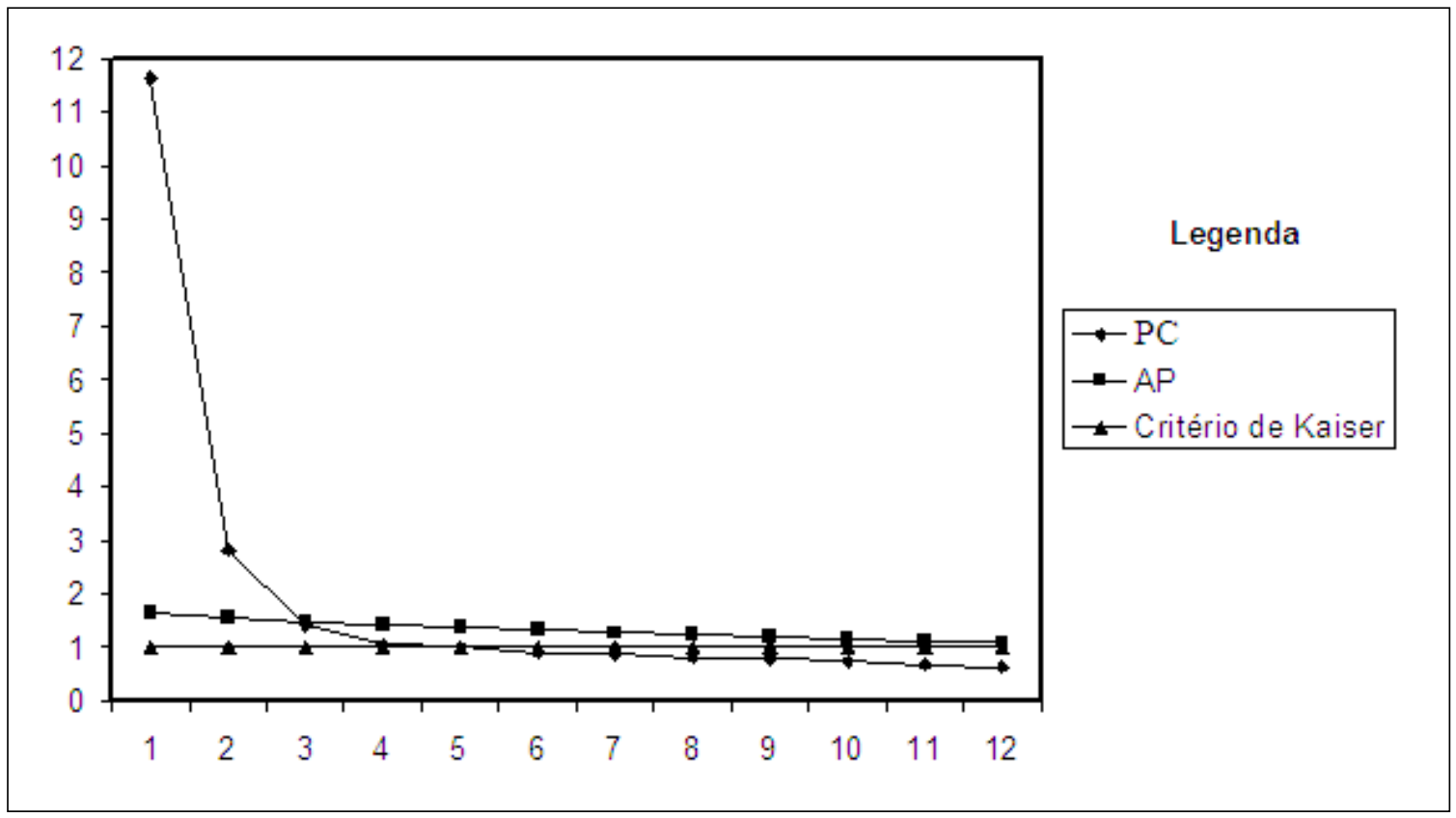

Figura 1. Distribuição gráfica dos valores próprios

De acordo com esta figura, até três componentes poderiam ser identificados a partir do critério de Cattell, cujos valores próprios foram 11,6, 2,8 e 1,4. Porém, a análise paralela revela a existência de no máximo dois componentes; o valor próprio do terceiro componente observado é inferior ao do valor médio desta análise $(1,5)$, denotando ser apropriado assumir uma estrutura bi-fatorial. Esta também é coerente com o modelo teórico que embasa a medida em questão e foi a observada no estudo de Rode (2005). Portanto, procedeu-se a uma nova análise $P C$, desta vez atendendo à recomendação de extrair dois componentes, definindo o método promax de rotação. Estes componentes explicaram conjuntamente $48,1 \%$ da variância total. Os resultados desta análise são mostrados na Tabela 1 .

Assumindo o critério de saturação mínima igual ou superior a $10,40 \mid$ em um único componente, primando por uma "estrutura simples", os dois componentes desta escala podem ser denominados como segue:

Componente I. Catorze itens integraram este componente, com saturações variando de 0,45 (tranqüilo) a 0,81 (entusiasmado); todos os demais itens expressaram afetos positivos (por exemplo, empolgado, animado, satisfeito, feliz). Portanto, a denominação deste componente parece clara, tratando-se de afetos positivos. Este apresentou um valor próprio (eigenvalue) de 10,6, explicando 38,7\% da variância total. Sua consistência interna, calculada por meio do Alfa de Cronbach $(\mu)$, foi 0,94, e homogeneidade média dos itens de 0,68 (correlação item-total média corrigida; amplitude de 0,54 a 0,80).
Componente II. Treze itens representaram este componente, cujas saturações variaram de -0,41 (calmo) a 0,77 (assustado); excetuando o item com menor saturação, que no caso foi negativa, todos os demais que formam este componente tiveram conotação negativa (por exemplo, desgostoso, depressivo, desencorajado, chateado). Neste caso, fica evidente sua etiqueta: afetos negativos. Seu valor próprio foi de 8,7, correspondendo à explicação de $9,4 \%$ da variância total, com $\mu$ de 0,87 . Em termos da homogeneidade dos seus itens, esta obteve um valor médio de 0,58 , variando de 0,29 a 0,75 .

Finalmente, uma nova análise PC, fixando a extração de um único componente, reuniu 27 dos 30 itens desta escala, ficando de fora os seguintes: assustado, ansioso e tímido, que obtiveram saturações inferiores a 10,401 . Este componente geral teve valor próprio de 11,6, explicando 38,7\% da variância total, com Alfa de Cronbach de 0,95. Sua homogeneidade foi de 0,61 , com valores dos itens variando de 0,42 (miserável) a 0,76 (satisfeito).

Em resumo, é possível identificar um componente geral e dois componentes específicos subjacentes à Escala de Afetos no Trabalho: afetos positivos e afetos negativos. Estes emergiram claramente, tomando-se em conta a distribuição gráfica dos valores próprios e a análise paralela. Os três índices de consistência interna, em torno de 0,90, alcançam os padrões recomendados. Portanto, em análises subseqüentes serão tidos em conta estes fatores específicos e o geral. 
Tabela 1

Estrutura Componencial da Escala de Bem-estar Afetivo no Trabalho

\begin{tabular}{|c|c|c|c|c|}
\hline \multirow[b]{2}{*}{ Item } & \multirow[b]{2}{*}{$\begin{array}{l}\text { Descrição } \\
\text { do Conteúdo }\end{array}$} & \multicolumn{3}{|c|}{ Componentes } \\
\hline & & $\mathrm{I}$ & II & $h^{2}$ \\
\hline 17 & Entusiasmado & $0,89 *$ & 0,15 & 0,81 \\
\hline 16 & Empolgado & $0,89 *$ & 0,13 & 0,81 \\
\hline 15 & Animado & $0,85^{*}$ & 0,02 & 0,72 \\
\hline 28 & Honrado & $0,83^{*}$ & 0,17 & 0,72 \\
\hline 29 & Satisfeito & $0,80 *$ & $-0,04$ & 0,64 \\
\hline 14 & Com energia & $0,78^{*}$ & 0,02 & 0,61 \\
\hline 27 & Agradecido & $0,77 *$ & 0,13 & 0,61 \\
\hline 23 & Feliz & $0,76^{*}$ & $-0,07$ & 0,58 \\
\hline 25 & Inspirado & $0,74 *$ & 0,05 & 0,55 \\
\hline 13 & Orgulho do que faço & $0,73^{*}$ & $-0,01$ & 0,53 \\
\hline 30 & À vontade & $0,72 *$ & 0,06 & 0,52 \\
\hline 09 & Contente & $0,64 *$ & $-0,19$ & 0,45 \\
\hline 06 & Alegre & $0,57^{*}$ & $-0,23$ & 0,38 \\
\hline 01 & Tranqüilo & $0,45^{*}$ & $-0,27$ & 0,27 \\
\hline 18 & Assustado & 0,25 & $0,77 *$ & 0,65 \\
\hline 11 & Desgostoso & $-0,09$ & $0,75^{*}$ & 0,57 \\
\hline 10 & Depressivo & 0,07 & $0,70^{*}$ & 0,49 \\
\hline 12 & Desencorajado & $-0,12$ & $0,69 *$ & 0,49 \\
\hline 03 & Chateado & 0,06 & $0,67 *$ & 0,45 \\
\hline 04 & Ansioso & 0,38 & $0,63^{*}$ & 0,54 \\
\hline 05 & Incomodado & $-0,09$ & $0,63 *$ & 0,40 \\
\hline 02 & Com raiva & $-0,06$ & $0,63 *$ & 0,40 \\
\hline 19 & Malsucedido & $-0,09$ & $0,63 *$ & 0,40 \\
\hline 21 & Triste & $-0,24$ & 0,58 & 0,39 \\
\hline 20 & Furioso & 0,15 & $0,54 *$ & 0,31 \\
\hline 08 & Confuso & 0,02 & $0,54 *$ & 0,29 \\
\hline 07 & Calmo & 0,32 & $-0,41^{*}$ & 0,27 \\
\hline 22 & Cansado & $-0,27$ & 0,23 & 0,13 \\
\hline 26 & Miserável & $-0,29$ & 0,18 & 0,12 \\
\hline 24 & Tímido & 0,09 & 0,28 & 0,09 \\
\hline \multicolumn{2}{|c|}{ Valor Próprio } & 10,6 & 8,7 & \\
\hline \multicolumn{2}{|c|}{ \% Variância Total } & 38,7 & 9,4 & \\
\hline \multicolumn{2}{|c|}{ Número de Itens } & 14 & 13 & \\
\hline \multicolumn{2}{|c|}{ Alfa de Cronbach } & 0,94 & 0,87 & \\
\hline
\end{tabular}

Nota: * carga fatorial considerada satisfatória, isto é, $\geq 10,40 \mid$.

\section{Afetos no Trabalho e Variáveis Demográficas}

Procurou-se primeiramente conhecer o tipo de afeto no trabalho que poderia descrever mais adequadamente a direção do bem-estar dos participantes da pesquisa. Neste sentido, calculou-se um teste $t$ para medidas repetidas (emparelhadas), observando-se uma diferença estatisticamente significativa, $t(282)=22,97, p<0,001$. Embora correlacionadas $(r=-0,57, p<0,001)$, a pontuação média nos afetos positivos $(M=3,82, D P=0,79)$ foi superior àquela observada para os afetos negativos $(M=2,01, D P=$ $0,70)$. Isso sugere que os participantes do estudo são em- pregados que gozam de bem-estar subjetivo no trabalho, mais do que mal-estar. Resta, entretanto, conhecer se estas pontuações variam em função de características demográficas dos participantes, especificamente do gênero e da idade.

Um passo inicial foi testar a influência destas duas variáveis com relação à pontuação total de afetos no trabalho. No caso, inverteram-se as escalas de respostas dos afetos negativos, sendo a maior pontuação indicação de maior bem-estar subjetivo (afetos positivos). Realizou-se uma MANOVA, considerando o gênero e a idade como variáveis antecedentes e a pontuação total em afetos no trabalho como critério. No caso da idade, considerando o critério prático da sua distribuição, criaram-se três categorias: até $21(27,9 \%)$, de 22 a $27(44,2 \%)$ e de 28 anos e mais $(27,9 \%)$. Nenhuma destas variáveis influenciou significativamente a pontuação total de bem-estar subjetivo (afetos) no trabalho, como se especifica: idade $[F(2,277)=2,31, p=0,07]$ e gênero $[F(1,277)=0,18, p>0,05]$.

Finalmente, procurou-se testar o efeito do gênero e da idade nos componentes de afetos positivos e negativos, tendo sido realizada uma MANOVA multi-fatorial. Também nestes casos, nenhuma das duas variáveis se mostrou preponderante para explicar a variação nos afetos entre os participantes do estudo, como segue: idade (Lambda de Wilks $=0,99, F=0,57, p=0,68$ ) e gênero (Lambda de Wilks $=0,99, F=1,34, p=0,26$ ).

\section{Discussão}

O objetivo principal deste estudo foi adaptar a JAWS para o contexto brasileiro, conhecendo evidências de sua validade fatorial e consistência interna, além de verificar se suas pontuações variam de acordo com a idade e o gênero dos participantes. Espera-se que este tenha sido alcançado. Não obstante, reconhecem-se algumas limitações potenciais da pesquisa que, embora não a invalidem ou comprometam os objetivos antes indicados, demandam pensar criticamente os resultados previamente descritos. Por exemplo, contou-se com uma amostra reduzida de participantes $(N<300)$, o que limita generalizar os resultados para o Brasil e mesmo o contexto paraibano. Contudo, não foi este o propósito do estudo, mas conhecer os parâmetros psicométricos desta escala. Neste sentido, a quantidade de participantes pode ser considerada suficiente (Watkins, 1989), permitindo que se discutam a seguir os principais resultados.

Inicialmente, cabe destacar que, diferentemente do que observaram os autores da versão original (Katwyk et al., 2000), não foram identificados quatro componentes, resultado do cruzamento das dimensões de prazer e excitabilidade: Alto Prazer - Alta Excitabilidade, Alto Prazer - Baixa Excitabilidade, Baixo Prazer - Alta Excitabilidade e Baixo Prazer - Baixa Excitabilidade. No presente estudo foi nítida a existência de dois grandes componentes, denominados de afetos positivos e afetos negativos, que foram também observados pelos autores previamente listados e que têm sido recentemente encontrados em pesquisa realizada na Eslovênia (Rode, 2005). 
Contudo, é importante que não se perca de vista que no estudo ora descrito e no deste autor foi empregado procedimento estatístico diferente daquele de Katwyk et al. (2000). Naquela oportunidade, realizou-se um escalonamento multidimensional, que permite mais facilmente visualizar relações estruturais entre componentes de duas ou mais dimensões, porém é menos criterioso e mais dado a interpretações e decisões subjetivas (Schiffman et al., 1981). Portanto, parece mais adequado comparar os resultados aqui descritos acerca da estrutura fatorial com aqueles apresentados por Rode (2005).

Rode (2005), considerando os 30 itens da JAWS, encontrou dois fatores (afetos positivos e afetos negativos) que, conjuntamente, explicaram 38,3\% da variância total. Considerando que cinco destes itens (tranqüilo, incomodado, orgulhoso, animado e empolgado) apresentaram baixa correlação item-total, decidiu realizar nova análise fatorial, retirando tais itens. Esta revelou os mesmos dois fatores, porém aumentou um pouco o montante de explicação da variância total, que passou a $42,2 \%$. No presente estudo foram encontrados claramente os mesmos fatores, porém explicando maior quantidade de variância total $(48,1 \%)$. Os índices de consistência interna para tais fatores no presente estudo foram ainda mais promissores que os de Rode (2005), aproximando-se dos descritos por Katwyk et al. (2000). De fato, foram idênticos no caso do fator específico de afetos positivos $(0,94)$ e fator geral de bem-estar afetivo $(0,95)$; apenas no fator de afetos negativos foi um pouco inferior $(0,87$ contra 0,92$)$. Portanto, parece adequado afirmar que existem evidências de validade fatorial e consistência interna deste instrumento no contexto brasileiro.

É comum relacionar fatores de bem-estar psicológico e subjetivo com variáveis demográficas, a exemplo do gênero (Caetano \& Estrada, 2006; Chaves et al., 2002; Fujita et al., 1991; Hutchinson et al., 2004; Peiró, 2006) e da idade (Otta \& Fiquer, 2004; Ryff, 1989; Santavirta et al., 2005). Embora existam mais evidências a favor de maior bemestar por parte de participantes do sexo masculino e mais velhos, como previamente ficou assinalado, isso não se constatou no presente estudo. Apesar de não ser possível uma explicação definitiva para esta independência dos afetos em relação à idade e ao gênero dos participantes desta pesquisa, é possível realizar algumas conjeturas: (a) estes compreenderam um grupo muito homogêneo em termos de idade, formado, principalmente, por jovens (cerca de $3 / 4$ têm até 27 anos). Isso, provavelmente, restringiu a possibilidade de encontrar qualquer diferença em termos de afetos; e (b) o gênero pode ser importante quando, atrelado a esta característica, existe uma diferenciação de status (Possati \& Dias, 2002). Na presente pesquisa, cerca de $71 \%$ dos participantes eram vendedores ou caixas, ou seja, gozavam do mesmo status ou papel social. Portanto, talvez se fossem consideradas pessoas de diferentes ocupações e responsabilidades laborais, as diferenças nas pontuações de afetos seriam mais evidentes em função do gênero e da idade.
Por fim, em termos de estudos futuros, ao menos três frentes deveriam merecer atenção: (a) poder-se-ia considerar uma amostra maior e mais diversificada de participantes, preferentemente de outras regiões do país. Nesta direção, talvez fosse pertinente considerar a aplicação deste instrumento entre profissionais da saúde (por exemplo, enfermeiras, médicos), reconhecidamente expostos a situações-limite que os têm levado a fadiga e estresse laboral (M. B. Carneiro \& Gouveia, 2004); (b) uma medida com 30 itens para cobrir unicamente dois fatores principais parece dispendiosa; mesmo que fossem quatro fatores, com um máximo de 20 itens seria suficiente, ficando cinco por cada fator. Portanto, caberia conhecer a adequação de abreviar a $J A W S$, que seria importante, sobretudo, considerando o contexto em que ela é costumeiramente aplicada: em organizações ou empresas. Nestes âmbitos é sempre útil contar com medidas breves, demandando tomar menos tempo dos trabalhadores; e (c) apesar de ter sido dado aqui um passo importante para adaptar esta escala para o contexto brasileiro, resta ainda conhecer a viabilidade de serem encontradas os quatro fatores preconizados por Katwyk et al. (2000). Sua estrutura parece evidenciar um circumplex, comum para descrever as relações dinâmicas e complexas que se estabelecem em relação aos afetos, traços de personalidade e valores, permitindo que se comprove através de modelagem por equações estruturais - SEM (ver Schwartz $\&$ Boehnke, 2004). Por exemplo, isso pode ser avaliado através da rotina CIRCUM, desenvolvida a partir de princípios da SEM (Browne, 1992, 1995).

\section{Referências}

Albuquerque, A. S., \& Tróccoli, B. T. (2004). Desenvolvimento de uma escala de bem-estar subjetivo. Psicologia: Teoria e Pesquisa, 20(2), 153-164.

Álvaro, J. L. (1992). Desempleo y bienestar psicológico. Madri, España: Siglo XXI.

Biblioteca Virtual em Saúde. (2006). Bem-estar no trabalho / emoções no trabalho / afetos no trabalho. Retrieved March, 10, 2006, from http://www.bvs-psi.org.br/

Browne, M. W. (1992). Circumplex models for correlation matrices. Psychometrika, 57(4), 469-497.

Browne, M. W. (1995). CIRCUM. Notes on usage. Columbus, $\mathrm{OH}$ : Browne.

Carneiro, T. L., \& Carvalho, J. L. (2003). Trabalho em transição, saúde em risco [Resenha]. Psicologia: Teoria e Pesquisa, 19(2), 185-186.

Carneiro, M. B., \& Gouveia, V. V. (2004). O médico e o seu trabalho: Aspectos metodológicos e resultados do Brasil. Brasília, DF: Conselho Federal de Medicina.

Caetano, A., \& Estrada, R. (2006). O bem-estar subjetivo dos advogados. Retrieved February, 02, 2006, from http:// www.oab.pt/Publicações/Boletim/detalhe_artigo

Camman, C., Fichman, M., Jenkins, D., \& Klesh, J. (1979). The Michigan Organizational Assessment Questionnaire. Unpublished manuscript, University of Michigan.

Carter, S. D. (2004). Reexamining the temporal aspects of affect: Relationships between repeatedey measured affective state, subjective well-being, and affective disposition. Personality and Individual Differences, 36(3), 381-391. 
Chaves, S. S. S. (2003). Valores como preditores do bem-estar subjetivo. Dissertação de Mestrado não-publicada, Departamento de Psicologia, Universidade Federal da Paraíba, João Pessoa, PB.

Chaves, S. S. S., Gouveia, V. V., Gusmão, E. E. S., Santos, W. S., \& Araújo, J. M. A. (2002). O bem-estar subjetivo em função do sexo e da classe social. Trabalho apresentado no VI Conhecimento em Debate, João Pessoa, PB.

Cheng, S.-T. (2004). Endowment and constrast: The role of positive and negative emotions on well-being appraisal. Personality and Individual Differences, 37(5), 905-915.

Christopher, A. N., Kuo, S. V., Abraham, K. M., Noel, L. W., \& Linz, H. E. (2004). Materialism and affective well-being: The role of social support. Personality and Individual Differences, 37(3), 463-470.

Clark, A. E. (1997). Job satisfaction and gender: Why are women so happy at work? Labour Economics, 4(4), 341-372.

Comissão das Comunidades Européias. (2002). Adaptação às transformações do trabalho e da sociedade: Uma nova estratégia comunitária de saúde e segurança 2002-2006. Retrieved December, 10, 2005, from http://www.europa.eu.int/eur.lex/ lex/LexUriserv.htm

Daley, A. J., \& Parfitt, G. (1996). Good health - Is it worth it? Mood states, physical well-being, job satisfaction and absenteeism in members and nom-members of a British corporate health and fitness club. Journal of Occupational and Organizational Behavior, 69(2), 121-134.

Diener, E. (2000). Subjective well-being: The science of happiness and a proposal for a national index. American Psychologist, 55(1), 34-43.

Diener, E., Suh, E. M., Lucas, R. E., \& Smith, H. L. (1999). Subjective well-being. Three decades of progress. Psychological Bulletin, 125(2), 276-302.

Diener, E., Suh, E. M., \& Oishi, S. (1997). Recent findings on subjective well-being. Indian Journal of Clinical Psychology, 24(1), 25-41.

Donaldson, S., \& Blanchard, A. (1995). The seven health practices, well-being, and performance at work: Evidence for the value of reaching small and underserved worksites. Preventive Medicine, 24(3), 270-277.

Evans, O., \& Steptoe, A. (2002). The contribution of gender-role orientation, work factors and home stressors to psychological well-being and sickness absence in male and famale-dominated occupational groups. Social Science \& Medicine, 54(4), 481492.

Fujita, F., Diener, E., \& Sandvik, E. (1991). Gender differences in negative affect and well-being: The case for emotional intensity. Journal of Personality and Social Psychology, 61(3), 427-434.

Gouveia, V. V., Chaves, S. S. S., Oliveira, I. C. P., Dias, M. R., Gouveia, R. S., \& Andrade, P. R. (2003). A utilização do QSG12 na população geral: Estudo de sua validade de construto. Psicologia: Teoria e Pesquisa, 19(3), 241-248.

Gusmão, E. E. S. (2004). A hipótese da congruência vocacional: Considerações acerca dos valores humanos e bemestar subjetivo. Dissertação de Mestrado não-publicada, Departamento de Psicologia, Universidade Federal da Paraíba, João Pessoa, PB.

Hayton, J. C., Allen, D. G., \& Scarpello, V. (2004). Factor retention decidions in exploratory factor analysis: A tutorial on parallel analysis. Organizational Research Methods, 7(2), 191-205.

Herzberg, F. (1959). The motivation to work. New York: John Wiley.
Hutchinson, G., Simeon, D. T., Bain, B. C., Wyatt, G. E., Tucker, M. B., \& Lefranc, E. (2004). Social and health determinants of well being and life satisfaction in Jamaica. International Journal of Social Psychiatry, 50(1), 137-146.

Idaszak, J. R., \& Drasgow, F. (1987). A revision of the Job Diagnostic Survey: Elimination of a measurement artifact. Journal of Applied Psychology, 72(11), 69-74.

Jamal, M. (1999). Job stress, type-A behavior, and well-being: A cross-cultural examination. International Journal of Stress Management, 6(1), 57-67.

Jorge, J., Bosmia, H., Peter, R., \& Siegrist, J. (2000). Job strain, effort-reward imbalance and employee well-being: A large-scale cross-sectional study. Social Science and Medicine, 50(9), 1317-1327.

Katwyk, P. T. V., Spector, P. E., Fox, S., \& Kelloway, E. K. (2000). Using the job-related affective well-being scale (JAWS) to investigate affective responses to work stressors. Journal of Occupational Health Psychology, 5(2), 219-230.

Kirschenbaum, A., Oigenblick, L., \& Goldberg, A. I. (2000). Wellbeing, work environment and work accidents. Social Science and Medicine, 50(5), 631-639.

Mäkikangas, A., \& Kinnunem, U. (2003). Psychosocial work stressors and well-being: Self-esteem and optimism as moderators in a one-year longitudinal sample. Personality and Individual Differences, 35(3), 537-557.

Morin, E. M. (2001). Os sentidos do trabalho. ERA - Revista de Administração de Empresas, 41(3), 9-19.

Otta, E., \& Fiquer, J. T. (2004). Bem-estar subjetivo e regulação de emoções. Psicologia em Revista, 10(15), 144-149.

Peiró, A. (2006). Happiness, satisfaction and sócio-economic conditions: Some international evidence. Journal of SocioEconomics, 35(2), 348-365.

Porto, J. B., \& Tamayo, A. (2003). Escala de valores relativos ao trabalho: EVT. Psicologia: Teoria e Pesquisa, 19(2), 145-152.

Possati, I. C., \& Dias, M. R. (2002). Multiplicidade de papéis da mulher e seus efeitos para o bem-estar psicológico. Psicologia: Reflexão e Crítica, 15(2), 293-301.

Rode, N. (2005). Translation of measurement instruments and their reliability: Na example of job-related affective well-being scale. Metodoloski zvezki, 2(1), 15-26.

Ryan, R. M., \& Deci, E. L. (2001). On happines and human potentials: A review of research on hedonic and eudaimonic well-being. Annual Review of Psychology, 52(1), 141-166.

Ryff, C. D. (1989). Happiness is everything or is it? Explorations on the meaning of psychological well-being. Journal of Personality and Social Psychology, 57(6), 1069-1081.

Santavirta, N., Kovero, C., \& Soloviera, S. (2005). Psychosocial work environment, well-being anda emotional exhaustion. A study comparing five age groups of female workers within the human sector. International Congress Series, 1280(1), 130-135

Sauter, S. L., \& Hurrel, J. J., Jr., (1999). Occupational Health Psychology: Origins, content, and direction. Professional Psychology: Resarch and Practice, 30(2), 123-128.

Schiffman, S. S., Reynolds, M. L., \& Young, F. W. (1981). Introduction to multidimensional scaling. New York: Academic Press.

Schwartz, S. H., \& Boehnke, K. (2004). Evaluating the structure of human values with confirmatory factor analysis. Journal of Research in Personality, 38(3), 230-255.

Sevastos, P., Smith, L., \& Cordery, J. L. (1992). Evidence on the reliability and construct validity of Warr's (1990) well-being and mental health measures. Journal of Occupational and Organizational Behavior, 65(1), 33-49. 
Gouveia, V. V., Fonsêca, P. N., Lins, S. L. B., Lima, A. V. \& Gouveia, R. S. V. (2008). Escala de Bem-Estar Afetivo no Trabalho (Jaws): Evidências de Validade Fatorial e Consistência Interna.

Spector, P. E., \& Jex, S. M. (1998). Development of four selfreport measures of job stressors and strain: Interpersonal Conflict at Work Scale, Organizational Constraints Scale, Quantitative Workload Inventory and Psysical Symptoms Iventory. Journal of Occupational Health Psychology, 3(4), 356-367.

Tabachnick, B. G., \& Fidell, L. S. (2001). Using multivariate statistics. Northridge, CA: Califórnia State University.

Warr, P. (1987). Work, unemployment and mental health. Oxford, England: Oxford University Press.

Watkins, D. (1989). The role of confirmatory factor analysis in cross-cultural research. International Journal of Psychology, 24(6), 685-701

Watson, D., Clark, L., \& Tellegen, A. (1988). Development and validation of brief measures of positive and negative affect: The PANAS scales. Journal of Personality and Social Psychology, 54(6), 1063-1070.

Wright, T. A., \& Doherty, E. M. (1998). Organizational behavior "rediscovers" the role of emotional well-being. Journal of Organizational Behavior, 19(5), 481-485.

Zapf, D. (2002). Emotion work and psychological well-being: A review of the literature and some conceptual considerations. Human Resource Management Review, 12(3), 237-268. 